\title{
COOL-ARREST: Results from a Pilot Multicenter, Prospective, Single-Arm Observational Trial to Assess Intravascular Temperature Management in the Treatment of Cardiac Arrest
}

\author{
Kelly N. Sawyer, MD, MS,' Michael Mooney, MD,2 Gregory Norris, MD, \\ Thomas Devlin, MD, PhD, Justin Lundbye, MD, Pratik B. Doshi, MD, Jonathan Kyle Hewett, MD, \\ Alan T. Kono, MD, Jesse P. Jorgensen, MD, and Brian J. O'Neil, MD ${ }^{10}$
}

Targeted temperature management (TTM) is recommended postcardiac arrest. The cooling method with the highest safety and efficacy is unknown. The COOL-ARREST pilot trial aimed to evaluate the safety and efficacy of the most contemporary ZOLL Thermogard XP Intravascular Temperature Management (IVTM) system for providing mild TTM postcardiac arrest. This multicenter, prospective, single-arm, observational pilot trial enrolled patients at eight U.S. hospitals between July 28, 2014, and July 24, 2015. Adult ( $\geq 18$ years old), out-of-hospital cardiac arrest subjects of presumed cardiac etiology who achieved return of spontaneous circulation (ROSC) were considered for inclusion. Patients were excluded if (1) awake or consistently following commands after ROSC, (2) significant prearrest neurological dysfunction, (3) terminal illness or advanced directives precluding aggressive care, and (4) severe hemodynamic instability or shock. Patient temperature was maintained at $33.0^{\circ} \mathrm{C} \pm 0.3^{\circ} \mathrm{C}$ for a total of 24 hours followed by controlled rewarming $\left(0.1-0.2^{\circ} \mathrm{C} / \mathrm{h}\right)$. Logistic regressions were used to assess association of good functional outcome (modified Rankin Scale $\leq 3$ ) measured at the time of hospital discharge with shockable rhythm (yes/no), age, gender, race/ethnicity, lay-rescuer cardiopulmonary resuscitation, time to basic life support (minutes), time to ROSC (minutes), lactate ( $\mathrm{mg} / \mathrm{dL}$ ), and $\mathrm{pH}$ on admission. The ZOLL IVTM system was effective at inducing TTM (median time to target temperature from initiation, 89 minutes [interquartile range 42-155]). Adverse events most often included electrolyte abnormalities and dysrhythmias. Of patients surviving to hospital discharge, 16/20 patients had a good functional outcome. A total of 18 patients survived through 90-day follow-up, at which time 94\% (17/18) of patients had good functional outcome. The COOL-ARREST pilot trial demonstrates high safety and efficacy of the ZOLL Thermogard XP IVTM system in the application of mild TTM postcardiac arrest. This observational trial also revealed noteworthy variability in the management of postcardiac arrest patients, particularly with the use of early withdrawal of life-sustaining therapy.

Keywords: targeted temperature management, induced hypothermia, intravascular device, safety, efficacy

\section{Introduction}

T ARGETED TEMPERATURE MANAGEMENT (TTM) at a consistent temperature is recommended for comatose survivors of cardiac arrest (Callaway et al., 2015). Although controversy exists regarding the optimum target temperature (Bernard et al., 2002; Hypothermia after Cardiac Arrest Study [HACA], 2002; Nielsen et al., 2013), avoidance of both hyperthermia and fluctuations in temperature for at least 24 hours after return of spontaneous circulation (ROSC) is

\footnotetext{
${ }^{1}$ Department of Emergency Medicine, University of Pittsburgh School of Medicine, Pittsburgh, Pennsylvania.

${ }^{2}$ Minneapolis Heart Institute Foundation, Minneapolis, Minnesota.

${ }^{3}$ Department of Neurology, McLaren Health, Flint, Michigan.

${ }^{4}$ Department of Neurology, University of Tennessee College of Medicine, Chattanooga Center, Chattanooga, Tennessee.

${ }^{5}$ The Greater Waterbury Health Network, Waterbury, Connecticut.

${ }^{6}$ Division of Critical Care, Department of Emergency Medicine, McGovern Medical School, UT Health, Houston, Texas.

${ }^{7}$ Division of Cardiology, University of South Carolina School of Medicine, Palmetto Health, Columbia, South Carolina.

${ }^{8}$ Cardiovascular Medicine, Geisel School of Medicine at Dartmouth College, Dartmouth, New Hampshire.

${ }^{9}$ Division of Cardiology, Heart \& Vascular Institute, University of South Carolina School of Medicine—Greenville, Greenville, South Carolina.

${ }^{10}$ Department of Emergency Medicine, Wayne State University, School of Medicine, Detroit, Michigan.
} 
recommended as part of comprehensive postcardiac arrest care to improve functional outcome.

There is variation in TTM practice due to limited evidence regarding how to best induce and maintain a given target temperature, and control rewarming (Camp-Rogers et al., 2013). Early clinical practices utilized ice packs, cooling blankets, and/ or water blankets to induce and maintain TTM. These methods may allow unintentional overcooling and wide fluctuations in temperature (Merchant et al., 2006). Ambient measures, such as room or ventilator temperature, and patient factors, such as basal metabolic rate, may interfere with tight temperature control, even if using a closed-loop system (Vanlandingham et al., 2015).

There are several feedback-loop devices commercially available for temperature management, including warming or cooling from environmental exposures. The use of these devices, whether surface or intravascular, has become common for TTM postcardiac arrest. Most studies include mixed methods of TTM provision, including varied device generations. The question of which type of cooling method demonstrates the highest safety and efficacy remains unanswered. The ZOLL Thermogard XP machine provides up to $170 \mathrm{~W}$ of power compared with previous machines with maximum 120 W. Thus, the aim of the COOL-ARREST pilot trial was to evaluate the safety and efficacy of the ZOLL Thermogard XP Intravascular Temperature Management (IVTM) system to induce, maintain, and reverse mild TTM postcardiac arrest.

\section{Materials and Methods}

This multicenter, prospective, single-arm, observational pilot trial enrolled patients at eight U.S. hospitals between July 28, 2014, and July 24, 2015. Adult (at least 18 years old), out-of-hospital cardiac arrest (OHCA) subjects of presumed cardiac etiology who achieved ROSC were considered for inclusion if informed consent was also provided by the patient's legally authorized representative within 12 hours of ROSC. There was no randomization or stratification scheme. This investigation was approved by each of the participating hospitals' institutional review boards. The study was overseen by an independent data safety monitoring board.

Patients were excluded from this investigation if (1) awake or consistently following commands after ROSC, (2) significant prearrest neurological dysfunction, (3) terminal illness or advanced directives precluding aggressive care, and (4) severe hemodynamic instability or shock, despite aggressive resuscitative efforts. Instability or shock was defined as ongoing hypotension despite adequate fluid resuscitation and two vasopressors. All patients followed their institutionspecific TTM protocol, utilizing the ZOLL Thermogard XP IVTM closed-loop cooling system (ZOLL, San Jose, CA) and strict hemodynamic monitoring in the intensive care unit.

\section{TTM protocol}

Temperature was measured preferentially by the esophagus but may have been initially by the bladder or rectum until an esophageal temperature probe was placed. Patient temperature was maintained at $33.0^{\circ} \mathrm{C} \pm 0.3^{\circ} \mathrm{C}$ for a total of 24 hours followed by controlled rewarming $\left(0.1-0.2^{\circ} \mathrm{C} / \mathrm{h}\right)$. Normothermia was maintained thereafter. Metabolic, ventilatory, and hemodynamic parameters were managed according to goal-directed targets for the first 72 hours.

\section{Data collection and outcomes}

Data were collected from hospital and emergency medical service (EMS) records, including baseline demographic characteristics, EMS provision of prehospital cooling, and all relevant time intervals from time of arrest or 911 call to hospital discharge or death, according to Utstein recommendations (Cummins et al., 1991). During the hospital stay, patient vital signs, laboratory results, and medications were recorded at strict intervals during TTM through hospital day 7, discharge, or death. Modified Rankin Scale (mRS) scores (Uyttenboogaart et al., 2005) were recorded at hospital discharge and at 90-day follow-up as secondary outcome measures. All patients were included in an intention-to-treat analysis.

The primary outcome was the proportion of subjects in which the IVTM system could therapeutically cool subjects to a target temperature of $33^{\circ} \mathrm{C} \pm 0.3^{\circ} \mathrm{C}$ (as measured by the temperature probe) and maintain it for 24 hours. Secondary objectives included patient safety (observed adverse events [AEs] up to 90 days), retention (ability to enroll and complete study protocol and time to complete enrollment), and functional outcome (mRS). All AEs that occurred through completion of the final follow-up visit, whether observed by the investigator or by the subject, and if thought to be devicerelated, were reported in detail on the appropriate case report form and followed to resolution or the end of study participation. Potential major AEs included catheter-related injury (arteriovenous fistula, bleeding related to the catheter requiring $\geq 1 \mathrm{U}$ of blood, clinically significant hematoma at catheter site requiring intervention, deep vein thrombosis unilateral to the catheter in place, known embolism [air, thrombus, and catheter fragment], nerve injury, pseudoaneurysm, vascular perforation, or dissection) and infection, either local or systemic (pneumonia, sepsis, meningitis, visceral organ).

\section{Follow-up period and functional outcome}

Subjects discharged from the hospital underwent functional outcome assessment (mRS) and evaluation for AEs. Subjects were scheduled for a 90-day postenrollment follow- up visit, preferably in-person but possibly by telephone, to include repeat functional outcome and AE assessment. An individual's legal representative or medical caregiver was interviewed in place of the subject if he or she was unable to participate.

\section{Sample size and statistical analysis}

The sample size $(n=50)$ for this pilot study was determined based on primary endpoint considerations, assuming a performance rate of $85 \%$ or more. The primary analysis will be based on intention to treat.

Continuous variables, including age (years), initial temperature $\left({ }^{\circ} \mathrm{C}\right)$, and all time intervals (minutes or hours), are reported as means and standard deviations (SDs) or medians with interquartile ranges (IQRs); categorical variables, including functional outcome at hospital discharge and 90 days, are reported as counts and percentages. All temperature data are presented as percent of time that the patient's actual temperature, as measured by the esophageal temperature probe, was within $\pm 0.3^{\circ} \mathrm{C}$ of the set temperature. The D'Agostino and Pearson test was to assess the normality of data.

Univariable and multivariable logistic regression was used to assess association of good functional outcome $(\mathrm{mRS} \leq 3)$ 


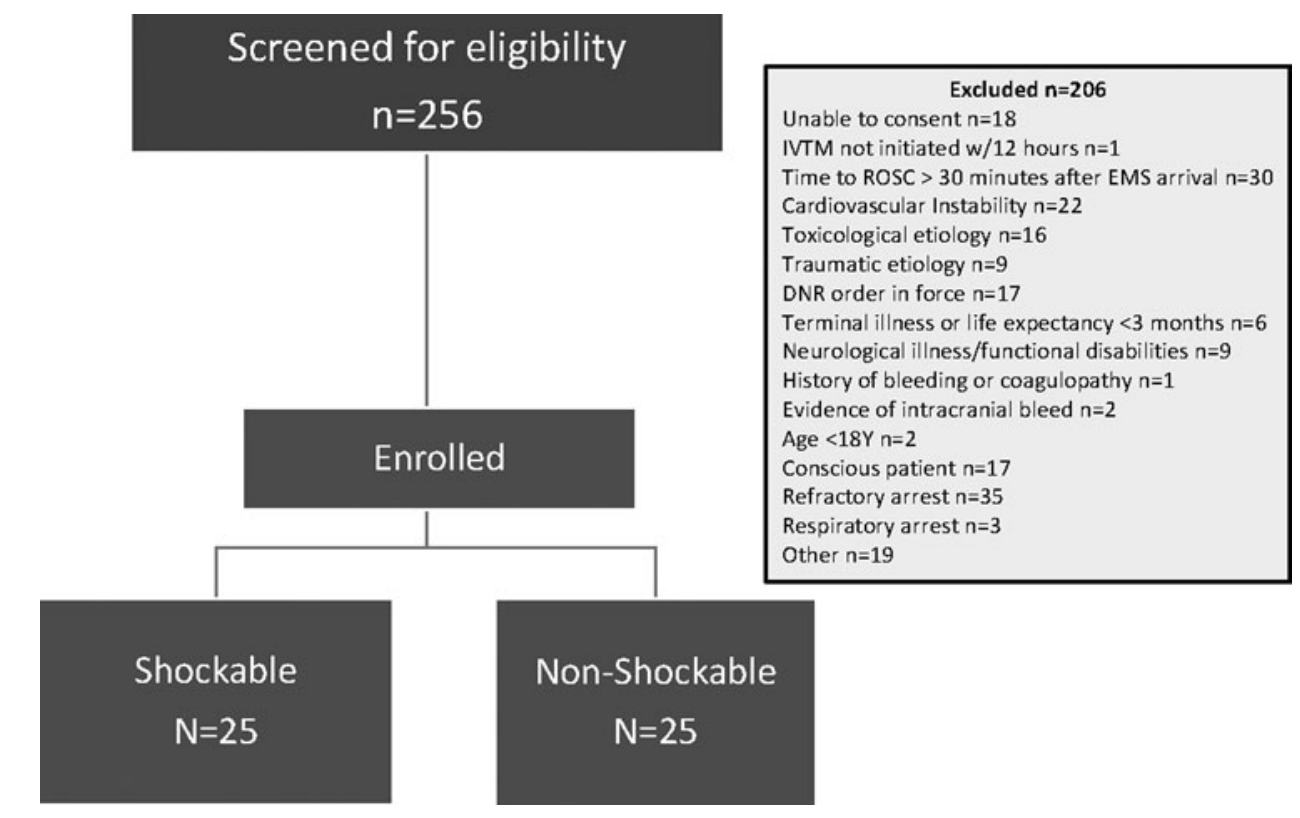

FIG. 1. Consort diagram of study population.

measured at the time of hospital discharge with shockable rhythm (yes/no), age, gender, race/ethnicity (Caucasian vs. other), lay-rescuer cardiopulmonary resuscitation (yes/no), time to basic life support (BLS) (minutes), time to ROSC (minutes), lactate $(\mathrm{mg} / \mathrm{dL})$, and $\mathrm{pH}$ on admission. Subjects were evaluated for potential poor prognosis utilizing the Cardiac Arrest Hospital Prognosis (CAHP) score (Maupain et al., 2016). Those with early withdrawal of life-sustaining therapy (WLST) were compared with those with WLST 24-72 hours and $>72$ hours utilizing medians and IQR of CAHP. Alpha of $<0.05$ was considered statistically significant. Data were analyzed with SAS (SAS Institute, NC) and Prism (GraphPad Software, CA).

\section{Results}

Our consort diagram is presented in Figure 1. Patient demographics, arrest characteristics, and treatment variables are summarized in Tables 1 and 2. A majority of subjects had OHCA witnessed by lay-rescuers $(82 \%)$ and occurred in a home $(56 \%)$ or public place $(20 \%)$. Only $44 \%$ received layrescuer CPR before EMS arrival.

All $(n=50)$ patients were successfully cooled to the target temperature. The ZOLL IVTM system was effective at inducing TTM (median time to target temperature from initiation, 89 minutes [IQR 42-155])). Most patients were cooled for less than 24 hours (median 22.7 hours, IQR 20.0-23.6). Rewarming occurred over a median of 13.5 hours (IQR 9.214.8) and normothermia was maintained for a median of 7.4 hours (IQR 4.9-10.2).

A total of 47 patients completed the hypothermia phase; 3 patients were excluded due to death before this stage. Subjects remained within $\pm 0.3^{\circ} \mathrm{C}$ of set temperature, median $98 \%$ of the time (IQR 94-100\%). A total of 46 patients completed the rewarming phase, due to 1 death before completing this stage. Subjects remained within $\pm 0.3^{\circ} \mathrm{C}$ of set temperature, median $99 \%$ of the time (IQR 86-100\%). Finally, a total of 40 subjects completed the normothermia maintenance phase; 6 patients were excluded due to death before completing this stage.
Subjects remained within $\pm 0.3^{\circ} \mathrm{C}$ of set temperature median $100 \%$ of the time. Figure 2 depicts median temperature data for the TTM treatment duration for the study cohort, demonstrating efficient achievement of target temperature and tight temperature control throughout the TTM period.

Regarding secondary outcomes, the safety profile was reasonable with no increase in AEs, while the IVTM was in place compared to any time during the study period. AEs are summarized in Supplementary Table S1. Total time from first enrollment through last patient follow-up was 364 days. Of patients surviving to hospital discharge, 16/20 patients had a good functional outcome. A total of 18 patients survived through 90-day follow-up. At the 90-day follow-up, $94 \%(17 / 18)$ of patients had good functional outcome (Table 3).

\section{Association of good functional outcome with baseline and treatment characteristics}

Initial shockable rhythm is highly significantly associated with good functional outcome $(p<0.001)$. Of the 50 subjects, there were 25 in the "shockable" category and 25 in the "nonshockable" category. Of the 25 nonshockable subjects, only $1 / 25(4 \%)$ had a good functional outcome at hospital discharge. In comparison, 15/25 (60\%) shockable subjects had a good outcome at discharge.

Regarding the other variables considered, lactate $(p=0.049)$ and $\mathrm{pH}(p=0.023)$ were statistically significantly related to functional outcome at hospital discharge; time to BLS ( $p=$ $0.052)$ and time to $\operatorname{ROSC}(p=0.063)$ were marginally related to functional outcome at hospital discharge; and there were no statistically significant associations observed with age, gender, race/ethnicity, lay-rescuer CPR, or automated external defibrillator. In multivariable modeling, after adjusting for the effect of initial shockable rhythm, no other covariates were significantly associated with the outcome variable. Supplementary Table S2 shows the association of good functional outcome at hospital discharge with baseline and treatment characteristics. 
Table 1. Patient Baseline Characteristics

\begin{tabular}{|c|c|}
\hline Characteristic & Summary \\
\hline Age, mean (SD) & $58.1(15.0)$ \\
\hline \multicolumn{2}{|l|}{ Gender } \\
\hline Male, $n(\%)$ & $27(54)$ \\
\hline Female, $n(\%)$ & $23(46)$ \\
\hline \multicolumn{2}{|l|}{ Race } \\
\hline Black, $n(\%)$ & $14(28)$ \\
\hline Caucasian, $n(\%)$ & $35(70)$ \\
\hline Other, $n(\%)$ & $1(2)$ \\
\hline \multicolumn{2}{|l|}{ Witnessed by lay-rescuer $(n=50)$} \\
\hline Yes, $n(\%)$ & $41(82)$ \\
\hline No, $n(\%)$ & $9(18)$ \\
\hline \multicolumn{2}{|l|}{ Location of arrest $(n=50)$} \\
\hline Home, $n(\%)$ & $28(56)$ \\
\hline Nursing care facility, $n(\%)$ & $2(4)$ \\
\hline Public place, $n(\%)$ & $20(40)$ \\
\hline \multicolumn{2}{|l|}{ Treated before EMS arrival $(n=50)$} \\
\hline Yes, $n(\%)$ & $23(46)$ \\
\hline No, $n(\%)$ & $27(54)$ \\
\hline \multicolumn{2}{|l|}{ Before EMS arrival $(n=50)$} \\
\hline Lay-rescuer ventilation, $n(\%)$ & $20(40)$ \\
\hline $\begin{array}{l}\text { Lay-rescuer chest compression, } \\
n(\%)\end{array}$ & $22(44)$ \\
\hline $\begin{array}{l}\text { Lay-rescuer defibrillation } \\
\quad(\mathrm{AED}), n(\%)\end{array}$ & $8(16)$ \\
\hline $\begin{array}{l}\text { Other (intubation, medication, } \\
\text { BLS), } n(\%)\end{array}$ & $3(6)$ \\
\hline \multicolumn{2}{|l|}{ ROSC before EMS arrival $(n=50)$} \\
\hline Yes, $n(\%)$ & $2(4)$ \\
\hline No, $n(\%)$ & $48(96)$ \\
\hline \multicolumn{2}{|l|}{ EMS treatment before hospital $(n=50)$} \\
\hline CPR, $n(\%)$ & $47(94)$ \\
\hline Defibrillation, $n(\%)$ & $27(55)$ \\
\hline Medication, $n(\%)$ & $36(72)$ \\
\hline \multicolumn{2}{|l|}{ Initial rhythm $(n=50)$} \\
\hline Ventricular fibrillation, $n(\%)$ & $15(30)$ \\
\hline Ventricular tachycardia, $n(\%)$ & $1(2)$ \\
\hline AED shock advised, $n(\%)$ & $7(14)$ \\
\hline Pulseless electrical activity, $n(\%)$ & $15(30)$ \\
\hline Asystole, $n(\%)$ & $8(16)$ \\
\hline AED no shock advised, $n(\%)$ & $4(8)$ \\
\hline \multicolumn{2}{|l|}{$\begin{array}{l}\text { ROSC after EMS arrival; before arrival } \\
\text { to } \operatorname{ED~}(n=48)\end{array}$} \\
\hline Yes, $n(\%)$ & $30(63)$ \\
\hline No, $n(\%)$ & $18(37)$ \\
\hline Admission $\mathrm{pH}(n=49)$ & Mean 7.2 \\
\hline Admission lactate, $\mathrm{mg} / \mathrm{dL}(n=29)$ & $\begin{array}{c}\text { (range } 6.80-7.46 \text { ) } \\
\text { Mean } 7.3 \\
\text { (range } 0.80-20.60 \text { ) }\end{array}$ \\
\hline
\end{tabular}

AED, automated external defibrillator; BLS, basic life support; CPR, cardiopulmonary resuscitation; ED, emergency department; EMS, emergency medical service; ROSC, return of spontaneous circulation; $\mathrm{SD}$, standard deviation.

\section{Withdrawal of life-sustaining treatment}

Overall, 32/50 patients died, most often related to brain death (18/32). Other causes included cardiac (6/32), respiratory $(6 / 32)$, and multiorgan failure (2/32). Table 4 summarizes the characteristics of patients who died early ( $<72$ hours) versus late ( $>72$ hours) in the hospital. Patients who died $<72$ hours
TABle 2. Treatment Intervals (Median Q1-Q3 [MINUTES])

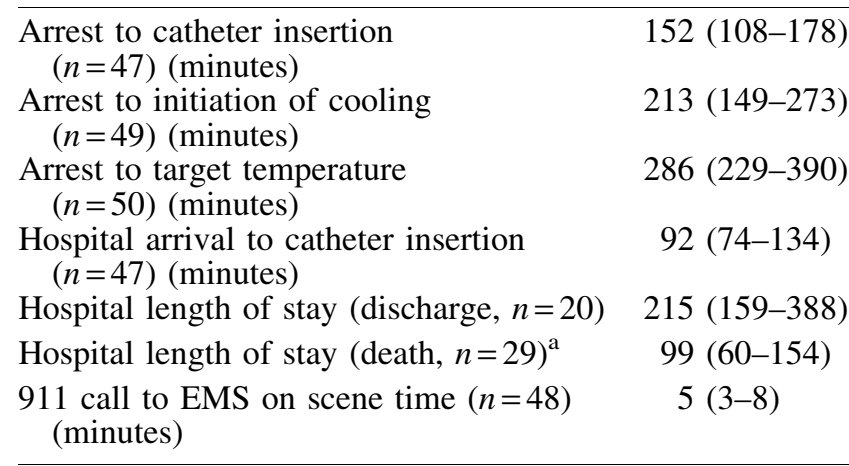

${ }^{\mathrm{a}}$ For within-hospital deaths.

from arrest tended to be a result of WLST due to "poor prognosis" secondary to encephalopathy, repeat cardiac arrest, and multiorgan failure. Those who died early also tended to be older. In both groups, $>90 \%$ had WLST due to "poor prognosis" assessment by clinical providers. Patients with WLST $<48$ hours from arrest had a higher risk of poor outcome with a CAHP score of 0.96, compared with patients with WLST 4872 hours (score 0.94 ) and those with WLST $>72$ hours (score 0.93) (Supplementary Table S3). Although the sample size is small, the CAHP score, which includes age, arrest setting, initial rhythm, time till ROSC, initial $\mathrm{pH}$, and epinephrine dose, does not appear to be a discriminator for WLST.

\section{Discussion}

The COOL-ARREST pilot trial demonstrates effective postcardiac arrest TTM induction, maintenance, and rewarming using the ZOLL Thermogard XP IVTM device. Patient temperatures were tightly controlled to a target temperature of $33^{\circ} \mathrm{C} \pm 0.3^{\circ} \mathrm{C}$ using this closed-loop intravascular system in all 50 patients enrolled. Overall, 36\% (18/50) survived to 90 days with few AEs observed throughout the study period. There was no increase in AEs while the IVTM system was in place. These data provide evidence to support the safety of the ZOLL IVTM device in the application of mild TTM to postcardiac arrest patients.

Our results are similar to the largest modern study evaluating TTM in postcardiac arrest patients (Nielsen et al., 2013). Target temperature was achieved quickly once the IVTM device was in place and turned on, although delays to intravascular device placement and system start-up did occur (Table 2). Furthermore, tight temperature maintenance and minimal deviation from target in our study (Fig. 2) are consistent with findings from patients treated with an intravascular device during the TTM trial (Glover et al., 2016). While on secondary analysis Glover et al. found no statistical difference between surface and intravascular device time to target temperature, they did find higher rates of temperature deviations with the surface devices. Similar to most studies evaluating the safety of TTM, AE rates were low in COOLARREST. Most often AEs included electrolyte abnormalities and dysrhythmias, similar to reports from other TTM studies (Bernard et al., 2002; HACA, 2002; Nielsen et al., 2013).

COOL-ARREST patients achieved target temperature more quickly than in the TTM trial (median 89 minutes [IQR 


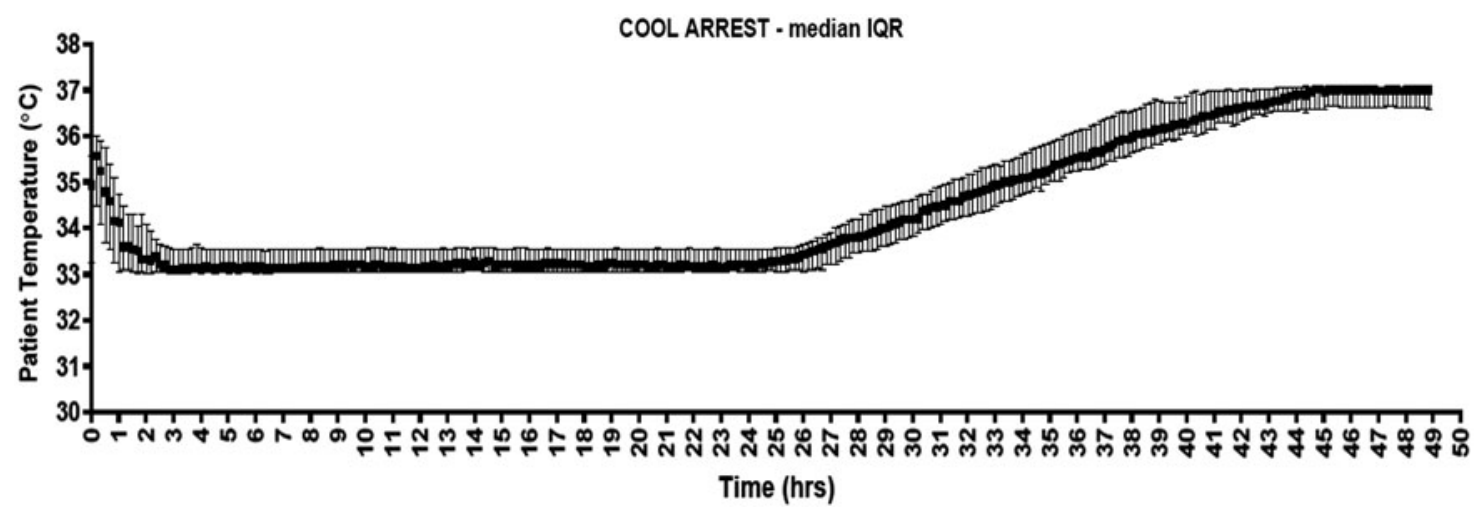

FIG. 2. Median patient temperature during study protocol.

42-155] vs. 210 minutes [IQR 180]) (data from Glover et al., 2016) and there were fewer deviations from set temperature. Patient factors (basal metabolic rate and body mass index), ambient room temperatures, or medication effects may have contributed to these differences. In addition, all patients in COOL-ARREST were treated with the ZOLL Thermogard $\mathrm{XP}$ device, whereas patients in previous studies, such as the TTM trial, were treated with a mixture of both surface and IVTM devices or a mixture of Thermogard XP with earlier IVTM products with lower effective cooling power. While preclinical studies demonstrate benefits from early TTM initiation and fast rates of TTM induction (Abella et al., 2004; Kim et al., 2016), results from observational clinical studies are controversial (Haugk et al., 2011; Sendelbach et al., 2012; Perman et al., 2015). Patients with more severe hypoxic brain injury may not resist being exposed to TTM and thus achieve target temperature more easily. Alternatively, quicker TTM induction can lead to a shorter overall TTM dose, which may be associated with outcome in certain postcardiac arrest phenotypes (Sawyer et al., 2014; Kirkegaard et al., 2017). Future studies with appropriate power are needed to assess the relationship between TTM process intervals and functional outcome.

Although our small sample size makes it problematic to draw any other conclusions, we observed a very large and statistically significant association between presenting with a shockable rhythm and outcome. Unexpectedly, we also observed a large rate of WLST before 72 hours from hospital admission (Table 4). Postcardiac arrest care guidelines have evolved to encourage withholding WLST for at least 72 hours after rewarming from TTM (Callaway et al., 2015), given several observational studies demonstrating delayed awakening in patients treated with TTM (Grossestreuer et al., 2013; Mulder et al., 2014; Elmer et al., 2016; Zanyk-McLean et al., 2017). Moreover, the influence of reperfusion injury,

Table 3. Functional Outcome at 90-Day FOLLOW-UP BY MRS

\begin{tabular}{lcr}
\hline & $m R S ~ 0-3$ & $m R S 4-6$ \\
\hline Shockable & $16(64 \%)$ & $9(36 \%)$ \\
Nonshockable & $1(4 \%)$ & $24(96 \%)$ \\
All & $17(34 \%)$ & $33(66 \%)$ \\
\hline
\end{tabular}

mRS, modified Rankin Scale. end-organ dysfunction, and drug metabolism/elimination on awakening remains uncertain (Anderson et al., 2016). In our pilot, observational efficacy trial, there was no mandated protocol for neuro-prognostication, and thus, our observation regarding WLST reveals significant pragmatic variation in site and physician/treating team care, as well as room for hospital system improvements. Based on the CAHP scoring, classic prognosticators (i.e., age, nonshockable rhythm, time to BLS, time to ROSC, arrest location) for poor functional outcome do not appear to have influenced WLST decisions in this study.

\section{Limitations}

The primary aim for this pilot study was to evaluate the efficacy of the ZOLL Thermogard XP IVTM device for inducing and maintaining TTM in postcardiac arrest patients,

Table 4. Patients Dying In-Hospital: Early ( $<72$ Hours) Versus Late ( $\geq 72$ Hours) AFTER ADMISSION

\begin{tabular}{lcr}
\hline & $\begin{array}{c}\text { Death } \\
<72 \text { hours } \\
(\mathrm{n}=10)\end{array}$ & $\begin{array}{c}\text { Death } \\
72 \text { hours } \\
(\mathrm{n}=19)\end{array}$ \\
\hline $\begin{array}{l}\text { Mean age (SD) } \\
\text { Witnessed arrest, } n(\%)\end{array}$ & $\begin{array}{c}6.8(13.3) \\
8(80)\end{array}$ & $\begin{array}{c}58.9(12.6) \\
16(84)\end{array}$ \\
$\begin{array}{l}\text { Initial rhythm } \\
\text { Shockable, } n(\%)\end{array}$ & $3(30)$ & $4(21)$ \\
$\quad$ Nonshockable, $n(\%)$ & $7(70)$ & $15(79)$ \\
Mean time (minutes) & $35.7(24.9)$ & $29.3(14.0)$ \\
$\quad$ arrest to ROSC (SD) & & \\
$\begin{array}{l}\text { Cause of death, } n(\%) \\
\quad \text { Encephalopathy }\end{array}$ & $6(60.0)$ & $14(73.7)$ \\
$\quad$ Cardiac cause & $2(20.0)$ & $3(15.8)$ \\
$\quad$ Respiratory cause & $0(0.0)$ & $2(10.5)$ \\
$\quad$ Multiorgan failure & $2(20.0)$ & $0(0.0)$ \\
Poor prognosis as reason & $9(90)$ & $18(94.7)$ \\
$\quad$ for WLST, $n(\%)$ & $1(10)$ & $1(5.3)$ \\
Futility, instability, & & \\
$\quad$ rearrest, $n$ (\%) & & \\
\hline
\end{tabular}

Three patients excluded from table for death in the emergency department (1) or after discharge from hospital (2).

WLST, withdrawal of life-sustaining therapy. 
which made blinding care providers impossible. As with any observational study, we were unable to control for all possible confounders. However, this design also gives our study strength, increasing the generalizability of our results across multiple sites and clinicians.

The lack of associations between age, lay-rescuer CPR, and time to ROSC with outcome, typically significant in OHCA studies, may also be attributed to the small sample size. It is likely that our results are also biased by local postcardiac arrest care and WLST practices. Larger studies, standardized WLST practice, and more varied experience with IVTM systems may reveal additional safety and efficacy data.

\section{Conclusion}

The COOL-ARREST pilot trial demonstrates the high safety and efficacy of the ZOLL Thermogard XP IVTM system in the application of mild TTM postcardiac arrest. This observational trial also revealed noteworthy variability in the management of postcardiac arrest patients, particularly with the use of early withdrawal of life-sustaining therapy.

\section{Acknowledgments}

The authors acknowledge the study sites, data coordinators, clinical care providers, patients, and caregivers.

\section{Author Disclosure Statement}

This study was funded by a grant from ZOLL Corporation. Study institutions received funding to run the trial, and investigators had access to the data. ZOLL Corporation reviewed the article before publication. Dr. O'Neil is the principal investigator of the COOL-ARREST trial and on the ZOLL advisory and speaker boards. Dr. Mooney is also a member of the ZOLL advisory board. During the study period, Dr. Jorgensen received honoraria from Edwards Lifesciences, Abiomed, Janssen Pharmaceuticals, and Abbott Laboratories. Dr. Doshi was PI for a clinical trial sponsored by Vapotherm, Inc. No other author has a financial conflict to disclose.

\section{Supplementary Material}

Supplementary Table S1

Supplementary Table S2

Supplementary Table S3

\section{References}

Abella BS, Zhao D, Alvarado J, Hamann K, Vanden Hoek TL, Becker LB. Intra-arrest cooling improves outcomes in a murine cardiac arrest model. Circulation 2004;109:2786-2791.

Anderson KB, Poloyac SM, Kochanek PM, Empey PE. Effect of hypothermia and targeted temperature management on drug disposition and response following cardiac arrest: a comprehensive review of preclinical and clinical investigations. Ther Hypothermia Temp Manag 2016;6:169-179.

Bernard SA, Gray TW, Buist MD, Jones BM, Silvester W, Gutteridge G, Smith K. Treatment of comatose survivors of out-of-hospital cardiac arrest with induced hypothermia. N Engl J Med 2002;346:557-563.

Callaway CW, Donnino MW, Fink EL, Geocadin RG, Golan E, Kern KB, Leary M, Meurer WJ, Peberdy MA, Thompson TM, Zimmerman JL. Part 8: post-cardiac arrest care: 2015
American Heart Association guidelines update for cardiopulmonary resuscitation and emergency cardiovascular care. Circulation 2015;132(18 Suppl 2):S465-S482.

Camp-Rogers TR, Sawyer KN, McNicol DR, Kurz MC. An observational study of patient selection criteria for postcardiac arrest therapeutic hypothermia. Resuscitation 2013; 84:1536-1539.

Cummins RO, Chamberlain DA, Abramson NS, Allen M, Baskett PJ, Becker L, Bossaert L, Delooz HH, Dick WF, Eisenberg MS, et al. Recommended guidelines for uniform reporting of data from out-of-hospital cardiac arrest: the Utstein Style. A statement for health professionals from a task force of the American Heart Association, the European Resuscitation Council, the Heart and Stroke Foundation of Canada, and the Australian Resuscitation Council. Circulation 1991;84:960-975.

Elmer J, Torres C, Aufderheide TP, Austin MA, Callaway CW, Golan E, Herren H, Jasti J, Kudenchuk PJ, Scales DC, Stub D, Richardson DK, Zive DM; Resuscitation Outcomes Consortium. Association of early withdrawal of life-sustaining therapy for perceived neurological prognosis with mortality after cardiac arrest. Resuscitation 2016;102:127-135.

Glover GW, Thomas RM, Vamvakas G, Al-Subaie N, Cranshaw J, Walden A, Wise MP, Ostermann M, Thomas-Jones E, Cronberg T, Erlinge D, Gasche Y, Hassager C, Horn J, Kjaergaard J, Kuiper M, Pellis T, Stammet P, Wanscher M, Wetterslev J, Friberg H, Nielsen N. Intravascular versus surface cooling for targeted temperature management after out-of-hospital cardiac arrest-an analysis of the TTM trial data. Crit Care 2016;20:381.

Grossestreuer AV, Abella BS, Leary M, Perman SM, Fuchs BD, Kolansky DM, Beylin ME, Gaieski DF. Time to awakening and neurologic outcome in therapeutic hypothermia-treated cardiac arrest patients. Resuscitation 2013;84:1741-1746.

Haugk M, Testori C, Sterz F, Uranitsch M, Holzer M, Behringer W, Herkner H; Time to Target Temperature Study Group. Relationship between time to target temperature and outcome in patients treated with therapeutic hypothermia after cardiac arrest. Crit Care 2011;15:R101.

Hypothermia after Cardiac Arrest Study. Mild therapeutic hypothermia to improve the neurologic outcome after cardiac arrest. N Engl J Med 2002;346:549-556.

Kim T, Paine MG, Meng H, Xiaodan R, Cohen J, Jinka T, Zheng H, Cranford JA, Neumar RW. Combined intra- and post-cardiac arrest hypothermic-targeted temperature management in a rat model of asphyxial cardiac arrest improves survival and neurologic outcome compared to either strategy alone. Resuscitation 2016;107:94-101.

Kirkegaard H, Søreide E, de Haas I, Pettilä V, Taccone FS, Arus U, Storm C, Hassager C, Nielsen JF, Sørensen CA, Ilkjær S, Jeppesen AN, Grejs AM, Duez CHV, Hjort J, Larsen AI, Toome V, Tiainen M, Hästbacka J, Laitio T, Skrifvars MB. Targeted temperature management for 48 vs 24 hours and neurologic outcome after out-of-hospital cardiac arrest: a randomized clinical trial. JAMA 2017;318:341-350.

Maupain C, Bougouin W, Lamhaut L, Deye N, Diehl JL, Geri G, et al. The CAHP (Cardiac Arrest Hospital Prognosis) score: a tool for risk stratification after out-of-hospital cardiac arrest. Eur Heart J 2016;37:3222-3228.

Merchant RM, Abella BS, Peberdy MA, Soar J, Ong ME, Schmidt GA, Becker LB, Vanden Hoek TL. Therapeutic hypothermia after cardiac arrest: unintentional overcooling is common using ice packs and conventional cooling blankets. Crit Care Med 2006;34(12 Suppl):S490-S494. 
Mulder M, Gibbs HG, Smith SW, Dhaliwal R, Scott NL, Sprenkle MD, Geocadin RG. Awakening and withdrawal of life-sustaining treatment in cardiac arrest survivors treated with therapeutic hypothermia*. Crit Care Med 2014;42:2493-2499.

Nielsen N, Wetterslev J, Cronberg T, Erlinge D, Gasche Y, Hassager C, Horn J, Hovdenes J, Kjaergaard J; TTM Trial Investigators. Targeted temperature management at $33^{\circ} \mathrm{C}$ versus $36^{\circ} \mathrm{C}$ after cardiac arrest. N Engl J Med 2013;369: 2197-2206.

Perman SM, Ellenberg JH, Grossestreuer AV, Gaieski DF, Leary M, Abella BS, Carr BG. Shorter time to target temperature is associated with poor neurologic outcome in postarrest patients treated with targeted temperature management. Resuscitation 2015;88:114-119.

Sawyer KN, Kurz MC, Elswick RK Jr.; Virginia Commonwealth University ARCTIC Investigators. An investigation of a hypothermic to ischemic ratio in patients following out-ofhospital cardiac arrest presenting with a shockable rhythm. Ther Hypothermia Temp Manag 2014;4:72-78.

Sendelbach S, Hearst MO, Johnson PJ, Unger BT, Mooney MR. Effects of variation in temperature management on cerebral performance category scores in patients who received therapeutic hypothermia post cardiac arrest. Resuscitation 2012; 83:829-834.
Uyttenboogaart M, Stewart RE, Vroomen PC, De KJ, Luijckx GJ. Optimizing cutoff scores for the Barthel index and the modified Rankin scale for defining outcome in acute stroke trials. Stroke 2005;36:1984-1987.

Vanlandingham SC, Kurz MC, Wang HE. Thermodynamic aspects of therapeutic hypothermia. Resuscitation 2015;86: 67-73.

Zanyk-McLean K, Sawyer KN, Paternoster R, Shievitz R, Devlin $\mathrm{W}$, Swor R. Time to awakening is often delayed in patients who receive targeted temperature management after cardiac arrest. Ther Hypothermia Temp Manag 2017;7:95-100.

Address correspondence to:

Kelly N. Sawyer, MD, MS

Department of Emergency Medicine University of Pittsburgh School of Medicine 3600 Forbes Avenue Iroquois Building Suite 400A Pittsburgh, PA 15261

E-mail: ksawyer6@yahoo.com 